\title{
Comportamento do consumidor português de vinho biológico
}

\author{
The behavior of the portuguese organic wine consumers \\ Keylor Villalobos Moya ${ }^{10}$, Maria Raquel Lucas² $^{2}$ \\ 'Escuela de Ciencias Agrarias, Facultad de la Tierra y el Mar, Universidad Nacional, Heredia, Costa Rica, \\ Keylor.villalobos.moya@una.cr \\ ²Departamento de Gestão, Escola de Ciências Sociais, Universidade de Évora e CEFAGE, Évora, Portugal, mrlucas@uevora.pt
}

Como citar: Moya, K. V. \& Lucas, M. R. (2021). Comportamento do consumidor português de vinho biológico. Revista de Economia e Sociologia Rural, 59(1), e238888. https://doi.org/10.1590/1806-9479.2021.238888

\begin{abstract}
Resumo: Embora nos últimos anos o vinho biológico tenha evoluído de forma positiva na produção e no consumo, devido a uma maior preocupação e consciência ambiental e com a saúde, continua a ser uma atividade económica em Portugal com um mercado muito reduzido, em comparação com outros países da União Europeia e com outros produtos biológicos de origem agroalimentar. Daí que o propósito do presente estudo tenha sido o de analisar o comportamento do consumidor em relação ao vinho biológico, testando variáveis como a perceção dos atributos do vinho biológico, o estilo de vida e a consciência com a saúde e a sensibilidade ao preço, para perceber a relação entre a atitude e a intenção de compra, consumo e recomendação de um vinho biológico. A metodologia adotada, de natureza conclusivo-descritiva, com levantamento de dados quantitativos a partir de um questionário online disponível durante três meses (março-maio 2019), permitiu determinar e explicar a importância da perceção dos atributos do vinho biológico, das atitudes e da consciência com a saúde na disposição de compra, consumo e recomendação de um vinho biológico.
\end{abstract}

Palavras-chave: comportamento do consumidor, fatores influenciadores, perfil do consumidor, vinho biológico.

\begin{abstract}
Although, in recent years, organic wine has evolved positively in production and consumption due to increased concern and environmental awareness and health, it remains an economic activity in Portugal with a very small market compared to other EU countries and with other organic products of agri-food origin. Hence, the purpose of this study was to analyze consumer behavior in relation to organic wine, testing variables such as the perception of the attributes of organic wine, lifestyle and health awareness and price sensitivity to understand the link between the attitude and the intention to buy, consume and recommend a biological wine. It was adopted the conclusive and descriptive methodology, with survey of quantitative data from an online questionnaire available for three months (March-May 2019), allowed to explain the importance of the perception of the attributes. Of organic wine, attitudes and health awareness in the willingness to buy, consume and recommend an organic wine.
\end{abstract}

Keywords: consumer behavior, consumers' profile, influencing factors, organic wine.

\section{Introdução}

O setor vitivinícola, estimulado pelas novas tendências de consumo, consciência com a saúde e aumento da "atitude verde" dos consumidores, tem-se ajustado e interessado pelas questões da sustentabilidade (ambiental, social, económica e institucional), e apostado no desenvolvimento de programas e divulgação de práticas sustentáveis e sua divulgação enquanto importantes ferramentas de marketing para a diferenciação e aceitação dos produtos e marcas no mercado (Sillani et al., 2017). Em relação à dimensão das empresas produtoras de vinho biológico em Portugal, não existem estatísticas oficiais disponíveis, embora seja provável que predominem as microempresas e pequenas empresas, em virtude 
de serem essas dimensões as que representam a maior parte dos operadores no setor do vinho em Portugal (Banco de Portugal, 2017). No ano de 2015, Portugal tinha 87 operadores com atividade de vinificação, existindo 5 organizações de produtores de vinho biológico no ano de 2016 (Direcção Geral de Agricultura e Desenvolvimento Rural, 2017). Apesar da importância da indústria do vinho em Portugal, pelo número de empresas e volume produzido (Banco de Portugal, 2017), pelo posicionamento na exportação, entre as maiores do mundo (Instituto da Vinha e do Vinho, 2017), pela forte cultura de consumo dos portugueses (Nielsen, 2017), o crescimento do mercado de vinho biológico não tem evoluído de forma ajustada, nem mostrado um impacto positivo no mercado nacional (Direcção Geral de Agricultura e Desenvolvimento Rural, 2017), como tem acontecido noutros países europeus produtores de vinho. Tal situação pode ser explicada pelo estudo realizado pela Direcção Geral de Agricultura e Desenvolvimento Rural (2017), sobre uma amostra dos operadores mais relevantes no mercado dos produtos biológicos, principalmente no comércio por grosso e retalhista e nalguns produtores com venda direta ao consumidor, para determinar o valor de vendas dos produtos biológicos. Relativamente aos produtos de origem vegetal, frutas e vegetais frescos, o valor de vendas obtido em cerca de um terço dos operadores foi superior a $€ 50000$. Quanto ao vinho biológico, o cenário foi pouco positivo, correspondendo ao grupo de alimentos com os valores mais baixos de vendas na maioria dos operadores, situando-se entre os 1000 e os $10000 €$. Alguns autores consideram a situação do mercado do vinho biológico idêntica à do mercado de produtos biológicos em geral, sustentando-a na pouca evolução do modo de produção biológico, no menor crescimento e atraso significativo deste modo de produção em comparação com outros países da UE, na reduzida dimensão do mercado, no preço elevado dos produtos biológicos ao consumidor e no desconhecimento destes sobre as vantagens e benefícios do seu consumo (Cabo et al., 2016). Também sobre o consumo de vinho biológico não existem, na atualidade, registos ou estatísticas oficiais. Apenas existe uma referência da Associação Interprofissional para a Agricultura Biológica no ano de 2010 mencionada por Rodrigues (2016), na qual se indica um consumo per capita anual de $2 €$, sendo este um valor muito baixo, em comparação com outros países (Holanda, Suécia, Italia e França), que, para esse período, consumiam, em média por ano, produtos biológicos num valor de $100 €$. Em outras fontes registam também valores idênticos de consumo para Portugal (Willer \& Lernoud, 2017), o que significa a existência de informação não atualizada e reflete, por um lado, a escassa pesquisa realizada sobre esta temática e, por outro, a necessidade de existir, de uma forma sistemática e continuada ao longo do tempo, informação sobre o mercado de vinho biológico. Daí que o objetivo deste trabalho tenha sido o de estudar o comportamento do consumidor português de vinho biológico, nomeadamente, compreender os fatores influenciadores do comportamento do consumidor na sua atitude e intenção de compra, consumo e recomendação do vinho biológico.

\section{Mercado e comportamento do consumidor de vinho biológico}

Principalmente impulsionados por mudanças nas escolhas, preferências e estilos de vida dos consumidores, alguns mercados de vinho biológico têm evoluído consideravelmente (Gilinsky et al., 2016). Existem cada vez mais consumidores preocupados e dispostos a experimentar e pagar preços premium por produtos saudáveis (Nielsen, 2018) que geram oportunidades para as empresas e induzem inovação, novas formas de diferenciação e adoção de estratégias sustentáveis (Jones \& Hill, 2010).

No contexto global, a Europa representava, em 2016, 88\% da área de produção mundial em modo biológico (Willer \& Lernoud, 2017) com uma produção de vinho biológico total estimada em 7,5 milhões de hectolitros em 2014, que representava apenas $2,8 \%$ da produção total de vinho (Le Douarin, 2016). Neste continente, o crescimento da atividade da vinha biológica resultou, fundamentalmente, dos efeitos das políticas adotadas na União Europeia e de uma crescente procura de mercado por bebidas biológicas (Centre for the Promotion of Imports, 2016). Contudo, embora cerca de $8,4 \%$ da totalidade da uva vindimada nesta região (328 000 hectares) seja produzida em modo biológico, nem toda a produção é usada para obter vinho biológico (Willer \& Lernoud, 2017). Segundo estes autores, os países com maior 
área destinada à produção em modo biológico de uva foram, no ano de 2016, a Espanha e a Itália, com mais de 100000 hectares, seguidos da França (com mais de 70000 hectares). Entre os principais mercados de vinho biológico da União Europeia encontram-se a França, Alemanha, Itália, Reino Unido e Áustria (Pink, 2015; Le Douarin, 2016). A Alemanha é o maior importador mundial de vinho biológico com um volume anual aproximado de 30 milhões de garrafas, o que equivale a $225 \mathrm{mil} \mathrm{hl}$ (Centre for the Promotion of Imports, 2016). As suas importações são oriundas de Espanha, Itália e França (Le Douarin, 2016). Outros importadores são a Noruega, Suécia, Finlândia, Dinamarca, Reino Unido, Suíça, Áustria e Países de Benelux (Centre for the Promotion of Imports, 2016).

Em Portugal, apesar de não existirem estatísticas sobre o mercado de vinho biológico, predominam as microempresas e pequenas empresas, de forma similar à maioria dos operadores no setor do vinho (Banco de Portugal, 2017). Alguns autores (Cabo et al., 2016; Willer \& Lernoud, 2017) consideram o mercado do vinho biológico com reduzida dimensão, preço elevado e desconhecimento das vantagens e benefícios do seu consumo por parte dos consumidores. Evidenciam ainda a escassa pesquisa sobre a temática e a necessidade de existir, de uma forma sistemática e continuada ao longo do tempo, informação sobre o mercado de vinho biológico português, nomeadamente, sobre o consumo, intenções e tendências de compra dos consumidores, muito útil para os responsáveis das empresas vitivinícolas tomarem as suas decisões de gestão e de marketing.

O comportamento do consumidor é um conceito fundamental ao marketing agroalimentar por as empresas procurarem alcançar os seus objetivos, satisfazendo as necessidades, os desejos e as expectativas dos consumidores. Esta orientação para o consumidor implica, por parte das empresas, o conhecimento do seu comportamento (Lucas, 2006). A abordagem interdisciplinar do comportamento do consumidor requer bons instrumentos de trabalho e de análise e a necessária integração de conhecimentos, pelo que os modelos de comportamento têm vindo a ser cada vez mais utilizados, com bons resultados. Distintos modelos do comportamento do consumidor podem ser encontrados na literatura da especialidade (Howard \& Sheth, 1969; Engel et al., 1995; Steenkamp,1997; Schiffman \& Kanuk, 2010), sendo um dos primeiros o de Nicosia (1966).

No caso concreto do vinho biológico, apenas um modelo explicativo do comportamento do consumidor de vinho foi encontrado na literatura. A sua análise permite identificar influências individuais do consumidor (perceções, atitudes, aspetos demográficos, estilo de vida, e personalidade e experiência), ambientais (como valores culturais, referência do grupo, ocasião de uso) e do produto (atributos do produto e nível de envolvimento) (Geraghty, 2010 apud Guia, 2014).

Entre as variáveis já investigadas e publicadas na literatura, o ter um estilo de vida saudável parece não ser um bom indicador para gerar boas atitudes em relação ao consumo do vinho biológico (Rojas-Méndez et al., 2015). A consciência com a saúde e com o ambiente influencia de forma positiva a atitude e a intenção de compra de vinho biológico (RojasMéndez et al., 2015; Basha et al., 2015), embora o fator saúde venha a ser considerado um melhor preditor para explicar a intenção de compra do que a preocupação ambiental (Asif et al., 2018; Yadav \& Pathak, 2016). A preocupação do consumidor com o ambiente pode ter um efeito preditor significativo no comportamento do consumidor, no que respeita à recomendação de um vinho biológico, mas não na sua intenção de compra (Kim \& Bonn, 2015). O nível de conhecimento do consumidor, pela influência na avaliação da sua qualidade, pode também ter um papel fundamental na escolha e compra de um vinho (Cardeira, 2009), havendo uma relação positiva entre conhecimento, procura do vinho biológico e estar mais disposto a comprar esse vinho (D'Amico et al., 2016). A sensibilidade ao preço, nomeadamente a perceção do preço premium em vinhos saudáveis e com menor teor de sulfitos, demonstrou estarem os consumidores espanhóis e italianos dispostos a pagar mais por esses vinhos, informação que foi útil para desenvolver uma nova estratégia para esses vinhos, com base no seu potencial efeito na diminuição das dores de cabeça pelo reduzido teor em sulfitos (Amato et al., 2017). As perceções positivas dos consumidores, face a produtos ambientalmente amigáveis, são bons indicadores da sua predisposição para a compra de alimentos biológicos, sendo os consumidores que priorizam esse tipo de produtos os mais propensos a comprar vinhos biológicos (Kim \& Bonn, 2015; Laroche et al., 2001; 
Shepherd et al., 2005; Barber et al., 2009). A confiança e a informação sobre o modo de produção são as principais motivações para a compra de um vinho biológico (Fotopoulos et al., 2003), sendo que o sabor do vinho biológico não favorece a intenção da sua compra (Rojas-Méndez et al., 2015). Também as atitudes positivas associadas aos produtos biológicos sustentam uma maior intenção de compra (Weisstein et al., 2014).

Na sua maioria, os estudos têm sido focados no comportamento do consumidor na intenção de compra de alimentos biológicos, sendo poucos os trabalhos de investigação que examinam modelos e fatores que influenciam o consumidor de vinho biológico. Na Tabela 1 , listam-se algumas dessas investigações, com os autores respetivos, identificando o objetivo abordado e as conclusões do estudo.

\section{Metodologia}

O presente trabalho caracteriza-se por usar um paradigma dedutivo e uma tipologia de investigação conclusiva-descritiva com abordagem quantitativa, com levantamento de dados de campo e análises estatísticas subjacentes. Face aos poucos estudos existentes sobre o mercado de vinho biológico em Portugal, desde a perspetiva do consumidor, considerou-se como base a investigação empírica realizada por Kim \& Bonn (2015) e Rojas-Méndez et al. (2015). A partir da investigação destes autores, foram consideradas três hipóteses (H. 1.a, H.1.b, H.1.c) úteis para avaliar a perceção dos atributos do vinho biológico como fator influenciador ou preditor (intenção a comprar, consumir e recomendar). Adicionalmente, foram ainda avaliados outros fatores influenciadores, relacionados com os aspetos individuais dos consumidores (consciência com a saúde H.2, estilo de vida saudável H.3), para determinar se esses fatores favoreciam ou não as atitudes do consumidor português face ao vinho biológico e também entender o poder preditivo da sensibilidade ao preço (H.4) e da atitude do consumidor (H.5) face ao vinho biológico (intenção de comprar, consumir e recomendar). A Figura 1 mostra as variáveis avaliadas e as hipóteses. Em síntese, as hipóteses avaliadas foram as seguintes:

Hipótese 1. A perceção do consumidor de vinho biológico sobre os atributos do vinho biológico tem uma influência significativa na disposição face ao vinho biológico (intenção de comprar, consumir e recomendar).

H. 1.a. A perceção do consumidor de vinho biológico relativo ao fator ambiente tem uma influência significativa sobre a disposição face ao vinho biológico.

H. 1.b. A perceção do consumidor de vinho biológico relativo ao fator confiança tem uma influência significativa sobre a disposição face ao vinho biológico.

H. 1.c. A perceção do consumidor de vinho biológico relativo ao fator sabor-valor tem uma influência significativa sobre a disposição face ao vinho biológico.

Hipótese 2. Quanto mais consciente for um consumidor em relação à saúde, mais favorável será a sua atitude para com o vinho biológico.

Tabela 1. Estudos sobre o comportamento do consumidor de alimentos e vinhos biológicos.

\begin{tabular}{|c|c|c|c|c|c|}
\hline $\begin{array}{l}\text { Tipo de } \\
\text { mercado } \\
\text { analisado }\end{array}$ & $\begin{array}{l}\text { Variáveis } \\
\text { analisadas }\end{array}$ & Objetivo do estudo & $\begin{array}{c}\text { Método de coleta de } \\
\text { dados }\end{array}$ & Principais conclusões & Autores \\
\hline \multirow{3}{*}{ Alimentos Bio } & $\begin{array}{l}\text { Intenção de compra, } \\
\text { valores pessoais. }\end{array}$ & $\begin{array}{l}\text { Investigar a relação entre } \\
\text { valores pessoais e } \\
\text { atitudes em um mercado } \\
\text { emergente }\end{array}$ & $\begin{array}{l}\text { Um questionário } \\
\text { aplicado nas feiras de } \\
\text { productos bio e outro } \\
\text { online. }\end{array}$ & $\begin{array}{c}\text { A atitude desempenha um papel } \\
\text { mediador entre valores pessoais e a } \\
\text { intenção de comprar esses produtos } \\
\text { no mesmo mercado. }\end{array}$ & $\begin{array}{c}\text { (Mainardes et al } \\
., 2017) .\end{array}$ \\
\hline & $\begin{array}{l}\text { Intenção de compra, } \\
\text { Atitude do } \\
\text { consumidor, } \\
\text { Perceção do } \\
\text { consumidor. }\end{array}$ & $\begin{array}{l}\text { Identificar a intenção de } \\
\text { compra de consumidores } \\
\text { em relação a alimentos } \\
\text { bio. }\end{array}$ & $\begin{array}{l}\text { Questionário aplicado } \\
\text { aos consumidores no } \\
\text { estabelecimento de } \\
\text { produtos bio. }\end{array}$ & $\begin{array}{l}\text { A qualidade dos produtos, a } \\
\text { preocupação com o meio ambiente, a } \\
\text { preocupação com a saúde e o estilo de } \\
\text { vida são motivos comumente para a } \\
\text { compra de alimentos bio. }\end{array}$ & $\begin{array}{c}\text { (Basha et al., } \\
\text { 2015). }\end{array}$ \\
\hline & $\begin{array}{c}\text { Atitudes, } \\
\text { Sustentabilidade. }\end{array}$ & $\begin{array}{l}\text { Estudar as atitudes e a } \\
\text { intenção dos } \\
\text { consumidores de } \\
\text { comprar alimentos }\end{array}$ & Questionário & $\begin{array}{c}\text { O atributo de bem-estar animal e o } \\
\text { atributo ambiental se correlacionam } \\
\text { positivamente com a intenção de } \\
\text { compra. }\end{array}$ & $\begin{array}{l}\text { (Ueasangkomsa } \\
\text { te \& } \\
\text { Santiteerakul, } \\
\text { 2016). }\end{array}$ \\
\hline
\end{tabular}


Tabela 1. Continuação...

$\begin{array}{ccccc}\text { Tipo de } & \text { Variáveis } & \text { Objetivo do estudo } & \begin{array}{c}\text { Método de coleta de } \\ \text { dados }\end{array} & \text { Principais conclusões } \\ \text { analisado } & \text { analisadas } & \text { Autores }\end{array}$

biológicos sob o princípio

do desenvolvimento sustentável.

Perceções do consumidor Disposição do consumidor, Qualidade.

Comportamento do consumidor, Consciência.

Preocupação ambiental Consciência saúde Teoria de comportamento planeado.

Práticas sustentáveis da cadeia de fornecimento, Confiança, Intenções comportamentais.

Lacuna atitudecomportamento, Escolha vinho, Comportamento consumidor.

Atributos vinho biológico,

Vinho Bio Conhecimento geral Intenções

comportamentais do consumidor.

Envolvimento vinho, Conhecimento do vinho, Consciência de saúde, Estilo de vida saudável, Consciência preço Atitude,

Comportamento.

\section{Pesquisa}

consumidor,

Mercado, Viticultura bio.
Fazer uma análise da literatura sobre a atitude

do consumidor em

relação aos alimentos bio.

Entender os fatores que afetam a intenção de compra de alimentos bio.

Questionário estruturado

nvestigar a intenção do consumidor de comprar alimentos bio no contexto de uma nação em desenvolvimento.

Estudar a perceção dos consumidores sobre práticas sustentáveis utilizadas por

fornecedores de vinho

biológico, e atitudes relativas aos atributos de vinho bio.

Explorar a lacuna atitude-comportamento e identificar barreiras para a compra de vinho bio

Examinar a relação entre as perceções dos consumidores sobre atributos do vinho bio.

Identificar os determinantes da compra de vinho bio.

Identificar as atitudes e expectativas dos consumidores sobre o vinho bio. em grupo

Entrevista a
Revisão de artigos publicados em revistas sobre consumidores de alimentos bio e agricultura sustentável entre 2000 e 2008.

Questionário aplicado consumidores, interceptados enquanto saíam de empresas como supermercados, lojas de bebidas e lojas de alimentos bio.

Painel de dados.

Questionário online bio, associada ao tema ambiental, não determina a disposição para comprar, mas existe uma correlação positiva nas perceções sobre os atributos do sabor e confiança com a disposição a comprar e recomendar.

Os principais preditores da compra de vinho bio são a atitude em relação ao vinho bio e o envolvimento no vinho. $A$ sensibilidade ao preço não foi encontrado para ser um bom preditor de compra de vinho bio.

O vinho bio tem uma imagem positiva em relação à produção de uvas e processamento de vinho, é percebido como mais saudável em comparação com o vinho convencional, no entanto, em relação à qualidade sensorial, o vinho bio ainda enfrenta problemas de imagem.
(Shafie \&

Rennie, 2012).

Asif et al 2018).

(Yadav \& Pathak, 2016)

(Bonn et al., 2016)

Schäufele \& Hamm, 2018)

(Kim \& Bonn 2015)

(Rojas-

Méndez et al., 2015)

(Stolz \& Schmidt, 2008). 
Hipótese 3 Quanto mais saudável for o estilo de vida do consumidor, mais favorável será a sua atitude para com o vinho biológico.

Hipótese 4. Quanto mais sensível for um consumidor ao preço, menor será a sua disposição para com o vinho biológico.

Hipótese 5. Quando a atitude do consumidor em relação ao vinho biológico é positiva, maior será a sua disposição face ao vinho biológico.
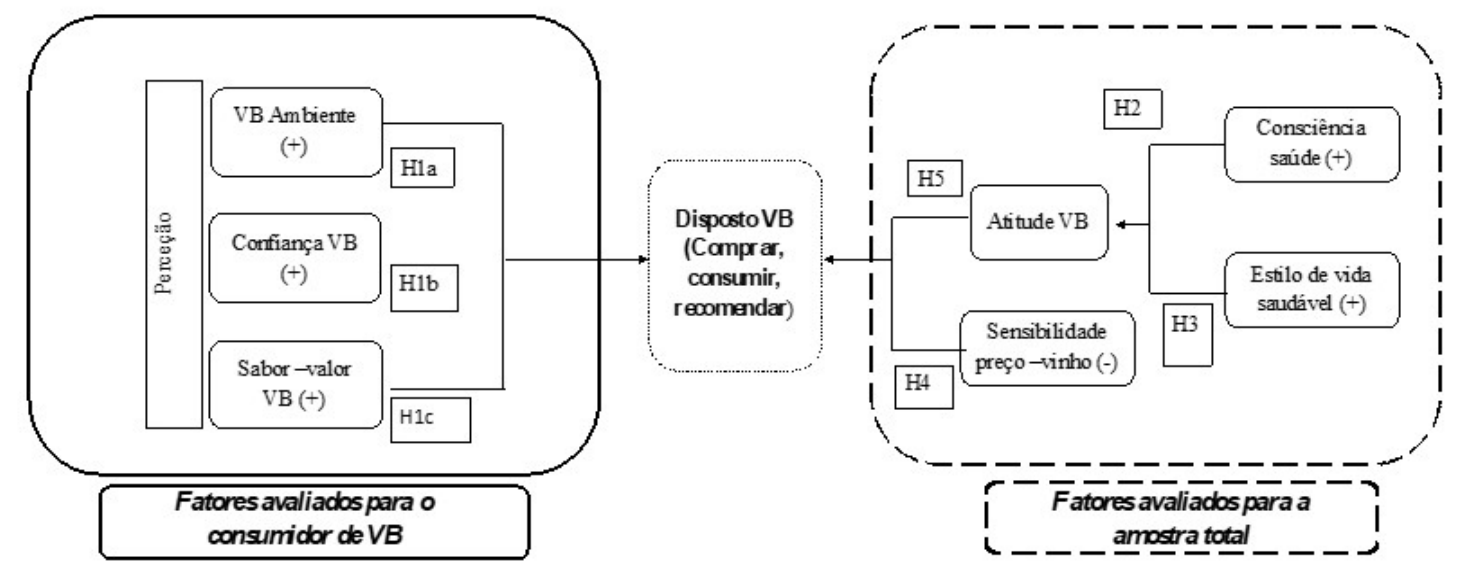

Figura 1. Hipóteses e variáveis avaliadas

Fonte: Adaptado de Kim \& Bonn (2015) e Rojas-Méndez et al. (2015)

A recolha de dados incluiu a aplicação de um questionário, o instrumento de pesquisa especificamente adaptado para o efeito. Seguindo os princípios da ética, foi garantida a confidencialidade das respostas e dos dados recolhidos. A elaboração do questionário foi feita na plataforma digital da Google Form e o seu link foi partilhado pelas redes sociais e por e-mail com mensagens explicativas do objetivo da investigação. Antes de ser partilhado, foi realizado um pré-teste online com 20 pessoas e feitos os ajustes necessários. Este pré-teste permitiu conhecer o tempo de preenchimento, a compreensão das questões e detetar erros e incongruências. O questionário, que se manteve ativo online durante três meses, de março a maio 2019, foi estruturado em quatro partes: i) Aspetos de qualificação e caracterização dos respondentes. Alguma destas questões cumpriram a função de caracterizar os consumidores de vinho, excluindo aqueles que não correspondiam ao desejado, perceber se os consumidores de vinho consumiram ou não vinho biológico e se estavam dispostos a recomendar, a comprar e a consumir o produto no curto prazo, este último item avaliado através de uma escala de Likert de 5 pontos (1 discordo totalmente e 5 concordo totalmente); ii) Itens sobre hábitos de compra e de consumo do vinho biológico; iii) Avaliação, através de uma escala de Likert, adaptada de Kim \& Bonn (2015) e de Rojas-Méndez et al. (2015), dos possíveis fatores influenciadores da disposição a recomendar, comprar e consumir o vinho biológico; iv) Caracterização sociodemográfica da amostra.

O universo da investigação foi o total dos elementos que tinham uma ou mais características comuns (Malhotra, 2011), ou seja, os portugueses residentes em Portugal, com idade superior a 18 anos e com hábitos de consumir vinho (sendo este um requisito obrigatório) e vinho biológico. A amostra foi construída através do método de amostragem não probabilístico, por conveniência, conhecido como "snowball", ou bola de neve (Silvério, 2000). Assim, foi solicitado aos elementos que contactavam com o questionário disponibilizado online, que o partilhassem pelos seus contatos e pelas pessoas que conheciam e com quem interagiam, que possuíssem as mesmas características. Este método, que foi escolhido pela facilidade e disponibilidade, baixo custo e rapidez, devido ao pouco tempo disponível, apresenta, contudo, o inconveniente de não ser possível generalizar os resultados e as conclusões ao universo, devido ao facto da amostra não ser razoavelmente representativa do mesmo (Malhotra, 2011; Kotler \& Armstrongs, 2007). A amostra foi 
composta por um total de 405 indivíduos, desagregados em duas categorias, consumidores de vinho e de vinho biológico, e consumidores de vinho que nunca consumiram vinho biológico.

Após tabulação dos dados, utilizando o programa estatístico SPSS (Statistical Package for Social Sciences versão 24 para o Windows 10), foi usada a técnica da análise fatorial (análises fatorial de componentes principais com rotação varimax) e a análise de regressão com procedimento stepwise para prever o comportamento do consumidor em relação aos fatores que podiam influenciar ou determinar as suas atitudes e a sua disposição (a comprar, consumir e recomendar) face ao vinho biológico. A técnica da análise fatorial (análise multivariada) consistiu em reduzir o número de variáveis de um conjunto de variáveis e identificar fatores com capacidade de influenciar as decisões do consumidor (Malhotra, 2011). Para tentar prever o comportamento do consumidor em relação aos fatores que podiam influenciar ou determinar as suas atitudes e a sua disposição (a comprar, consumir e recomendar) face ao vinho biológico, foi usada a análise de regressão. Esta técnica consiste em detetar os fatores com poder de prever o comportamento de uma variável quantitativa (variável dependente, endógena) a partir de uma ou mais variáveis quantitativas (variáveis independentes, exógenas, explicativas) (Pestana \& Gageiro, 2014).

\section{Análise e discussão dos resultados}

\subsection{Caracterização da amostra}

A partir do questionário online foi possível a recolha de 405 respostas, sendo excluídas, de acordo com os pressupostos estabelecidos, 46 respostas, por não serem consumidores de vinho, portugueses, ou residentes em Portugal. A amostra incluiu um total de 359 indivíduos consumidores de vinho, dos quais 197 já tinham consumido vinho biológico, e 162 nunca tinham consumido vinho biológico. Perto de metade tinha um grau de conhecimento intermédio de vinhos $(46,80 \%)$, uma terça parte era conhecedores novatos $(28,97 \%)$, uma parte menor de conhecedores avançados $(16,99 \%)$, sendo poucos os que se consideravam especialistas no tema do vinho (7,24\%). Afirmaram ter hábitos de consumo: diário $(21,17 \%)$, semanal (uma vez $28,13 \%$ ) ou bissemanal $(28,41 \%)$. Em relação ao género, a amostra distribuise de uma forma equilibrada, entre o masculino $(49,86 \%)$ e o feminino $(50,14 \%)$. A faixa de idade com maior quantidade de elementos foi a de $35-44$ anos $(28,13 \%)$, seguida da faixa $45-$ 54 e $25-34$, com quantidades similares (22,56\% e $21,45 \%$ respetivamente), havendo ainda inquiridos na faixa etária dos 18-24 (10,31\%), dos 55-64 (14,76\%) e maiores de 65 anos (2,79\%). Um pouco mais de cinquenta por cento apresentava habilitações literárias ao nível de licenciatura $(35,10 \%)$ ou mestrado $(21,45 \%)$, sendo a metade restante composta, principalmente, por pessoas com ensino secundário $(15,60 \%)$, pós-graduação $(11,70 \%)$ e curso profissional $(7,80 \%)$. Em termos de representação geográfica, a amostra continha residentes em todos os distritos de Portugal continental e da Região Autónoma dos Açores, sendo de destacar, com mais relevância, os distritos de Évora (40, 67\%), Lisboa (13, 93\%), Porto $(8,91 \%)$ e Setúbal $(6,13 \%)$.

\subsection{Fatores influenciadores do comportamento do consumidor}

\subsubsection{Perceção dos Atributos}

Como primeiro ponto para responder às hipóteses, realizou-se uma análise fatorial, para agrupamento das variáveis avaliadas. Foi usado o método de componentes principais para a extração dos fatores com rotação varimax. Foram retidos três fatores com valor próprio (eigenvalue) superior a 1 (Tabela 2), e retidas aquelas variáveis com cargas fatoriais (fator loading) superiores a 0,5 (dez de quinze variáveis foram retidas), para conformar três fatores: Ambiente, Confiança e Sabor-Valor, com uma variância de 20,746\%, 17,924\%, e 12,55\% respetivamente (Tabela 2). A estrutura fatorial resultante é muito semelhante ao modelo proposto por Kim \& Bonn (2015), com a diferença de que se obteve um fator apenas com dois 
itens com cargas fatoriais próximas, pelo que se considerou chamar ao terceiro fator saborvalor. Foi realizado o teste Kaiser-Meyer-Olkin (KMO) para verificar quão adequadas foram a aplicação e a validade da análise fatorial para o conjunto de variáveis. O valor de KMO obtido foi de 0,755 , o que indica uma boa adequação dos dados à análise fatorial (Dziuban \& Shirkey, 1974; Damásio, 2012; Pestana \& Gageiro, 2014).

Também a verificação da consistência interna de cada grupo de variáveis foi avaliada através da medida de Alpha de Cronbach, atingindo correlações entre 0,677 e 0,756. Os fatores ambiente e confiança apresentaram valores de Alpha Cronbach aceitáveis $(>0,7)$, mas o valor do fator sabor-valor foi relativamente fraco, mas próximo a 0,7 (Pestana \& Gageiro, 2014) (Tabela 2). Os índices de ajustamento GFI, AGFI-GFI apresentam valores de 0,93 e 0,87, respetivamente, sendo o RMSR de 0,04. Pestana \& Gageiro (2014) indicam que para valores de GFI entre 0,90 e 0,951, a análise fatorial é razoável, e para valores de RMSR $<0,05$, a análise é muito boa, pelo que se pode considerar a existência de um bom ajustamento, estando os dados muito bem adaptados à análise fatorial.

Tabela 2. Perceção dos atributos do vinho biológico

\begin{tabular}{|c|c|c|c|c|}
\hline Fatores e variáveis & $\begin{array}{l}\text { Fator } \\
\text { loading }\end{array}$ & Eigenvalue & $\begin{array}{l}\text { Variância } \\
\text { \% }\end{array}$ & $\begin{array}{l}\text { Cronbanch's } \\
\text { alpha }\end{array}$ \\
\hline $\begin{array}{l}\text { O vinho biológico só contém } \\
\text { ingredientes naturais. }\end{array}$ & 0,827 & \multirow{4}{*}{4,213} & \multirow{4}{*}{20,746} & \multirow{4}{*}{0,756} \\
\hline $\begin{array}{l}\text { A embalagem do vinho biológico é } \\
\text { amigável com o ambiente. }\end{array}$ & 0,735 & & & \\
\hline $\begin{array}{l}\text { O vinho biológico é produzido de } \\
\text { forma amiga do ambiente. }\end{array}$ & 0,729 & & & \\
\hline Fator 2. Confiança & & & & \\
\hline $\begin{array}{c}\text { É importante conhecer a empresa } \\
\text { vitivinícola produtora de vinhos } \\
\text { biológicos. }\end{array}$ & 0,871 & \multirow{6}{*}{2,050} & \multirow{6}{*}{17,924} & \multirow{5}{*}{0,737} \\
\hline $\begin{array}{l}\text { É importante conhecer o enólogo } \\
\text { responsável. }\end{array}$ & 0,737 & & & \\
\hline $\begin{array}{l}\text { É importante que o vinho biológico } \\
\text { seja produzido em Portugal. }\end{array}$ & 0,683 & & & \\
\hline $\begin{array}{l}\text { A qualidade do vinho biológico é o } \\
\text { mais importante. }\end{array}$ & 0,578 & & & \\
\hline $\begin{array}{l}\text { Quero aprender mais sobre os vinhos } \\
\text { biológicos. }\end{array}$ & 0,539 & & & \\
\hline \multicolumn{2}{|l|}{ Fator 3. Sabor e valor } & & & \\
\hline $\begin{array}{l}\text { O vinho biológico tem um sabor } \\
\text { agradável. }\end{array}$ & 0,633 & \multirow{2}{*}{1,436} & \multirow{2}{*}{12,655} & \multirow{2}{*}{0,677} \\
\hline $\begin{array}{l}\text { O vinho biológico é um bom valor } \\
\text { para o meu dinheiro. }\end{array}$ & 0,606 & & & \\
\hline
\end{tabular}

$\mathrm{Na}$ análise de regressão múltipla linear, foi possível detetar que todos os fatores independentes possuem uma correlação linear positiva significativa com a variável dependente (disposição face ao vinho biológico, Tabela 3), sempre que as restantes variáveis eram constantes. $O$ fator sabor-valor foi o que mostrou uma maior correlação linear positiva com a variável dependente (disposição face ao VB), com um coeficiente de regressão de $\beta=$ 1,227 (Tabela 3), pelo que este fator se considerou aquele que tem maior poder para prever a disposição dos consumidores face ao vinho biológico. No caso da pesquisa desenvolvida por Kim \& Bonn (2015), foram avaliados estes mesmos fatores, embora o principal fator influenciador da disposição face ao vinho biológico identificado tenha sido a confiança. $\mathrm{Na}$ Tabela 3, também se indicam os resultados que respondem às hipóteses testadas neste 
estudo, através da análise de regressão linear, sendo as variáveis ambiente $(\beta=0,750, p<$ $0,005)$, confiança $(\beta=0,564, p<0,005)$ e sabor-valor $(\beta=1,227, p<0,005)$ as que apresentaram significância na disposição face ao vinho biológico. Para o teste de coeficiente de regressão $\beta$ está associado um valor de significância (Sig(1-tailed)) de 0,01, 0,016 e 0,000 para os fatores ambiente, confiança e sabor-valor respetivamente (Tabela 3), o que significa que, ao ter uma significância de $p<0,05$, as hipóteses avaliadas (H1.a, H1.b e H1.c) são aceites.

Tabela 3. Influência da percepção na disposição face ao vinho biológico

\begin{tabular}{cccc} 
Fatores & \multicolumn{3}{c}{ Disposição face ao vinho biológico } \\
& Coef. regressão $\beta$ & Sig(1-tailed) & Hipótese \\
Ambiente & 0,750 & 0,01 & H1.a Aceita-se \\
Confiança & 0,564 & 0,016 & H1.b Aceita-se \\
Sabor-valor & 1,227 & 0,000 & H1c Aceita-se \\
& $R^{2} 0,294 / R^{2}$ Ajustado 0,276 & \\
\hline
\end{tabular}

H 1.a. A perceção do consumidor de vinho biológico sobre o fator ambiente tem uma influência significativa sobre a disposição face ao vinho biológico.

H 1.b. A perceção do consumidor de vinho biológico sobre o fator confiança tem uma influência significativa sobre a disposição face ao vinho biológico.

H 1.c. A perceção do consumidor de vinho biológico sobre o fator sabor-valor tem uma influência significativa sobre a disposição face ao vinho biológico.

Conseguiu-se, assim, verificar a importância destes três fatores para os portugueses, na disposição da compra, consumo e recomendação dos vinhos biológicos. Segundo o modelo de Kim \& Bonn (2015), para os americanos, o fator da perceção sobre os atributos do vinho biológico, associado ao tema ambiental, não determina a disposição para comprar, embora em outros estudos as perceções dos consumidores em relação aos benefícios ambientais estejam relacionados positivamente com a compra do vinho biológico (Bonn et al., 2016; Mann et al., 2012). Ainda assim, Kim \& Bonn (2015) determinaram uma correlação positiva nas perceções sobre os atributos do sabor e confiança com a disposição a comprar e recomendar um vinho biológico, indo ao encontro dos resultados encontrados na presente investigação.

Um dos principais problemas do marketing verde de hoje é a falta de confiança do consumidor na comunicação de informações ambientais por parte das empresas, que precisam conhecer mais os benefícios dos produtos biológicos e, também, confiar nos produtos e na cadeia de fornecimento (Daugbjerg et al., 2014; Nuttavuthisit \& Thøgersen, 2017); Pivato et al., 2008; Yin et al., 2010), convertendo-se este num aspeto importante e decisivo para a disposição a comprar. Aparentemente, esta situação repete-se no contexto do vinho biológico. Com o intuito de verificar se o modelo é adequado, foram feitas duas análises dos pressupostos da regressão linear múltipla (normalidade dos resíduos estandardizados e a multicolinearidade). Através do teste Shapiro Wilk foi testada a normalidade dos resíduos estandardizados, obtendo-se uma distribuição normal dos dados ( $p$-valor $>0,05$ ), cumprindo o pressuposto de normalidade dos resíduos. Não se detetou multicolinearidade entre os fatores exógenos $(V I F=1)$, o que indica a não existência de uma associação linear entre os fatores exógenos, o que cumpre o pressuposto do modelo de regressão linear das variáveis exógenas serem linearmente independentes

\subsubsection{Consciência com a saúde e estilo de vida saudável}

Com base em Rojas-Méndez et al. (2015), foi necessário avaliar a consistência interna dos dados de cada construto (agrupação de itens de cada fator, calculado pela média das variáveis de cada) a partir do teste Alpha de Cronbach, apresentando todos valores aceitáveis acima de 0,7 (Tabela 4), com exceção do fator de consciência com a saúde mas que se encontrava próximo a 0,7 . 
Tabela 4. Estatística descritiva para cada fator preditor

\begin{tabular}{cccc} 
Constructo & \# Itens & Média & Alpha Cronbach \\
Consciência com a saúde & 7 & 3,66 & 0,627 \\
Estilo de vida saudável & 14 & 3,43 & 0,784 \\
Atitude face vinho biológico & 9 & 3,23 & 0,723 \\
\hline
\end{tabular}

Para testar as hipóteses, foram usadas regressões múltiplas com o método stepwise. Uma das hipóteses a testar foi a predição entre uma maior consciência com a saúde e uma maior atitude para com o vinho biológico.

\section{H 2. Quanto mais consciente for um consumidor com a saúde, mais favorável será a sua atitude para com o vinho biológico.}

Deste modo, a hipótese $\mathrm{H} .2$ foi confirmada no modelo obtido ao ter um valor de significância $p<0,05$ (Tabela 5), convergindo estes com os resultados de outras investigações (Rojas-Méndez et al., 2015; Asif et al., 2018; Yadav \& Pathak, 2016). Os resultados obtidos permitiram detetar que o fator consciência com a saúde mostrou uma correlação linear positiva com a variável dependente (atitude para com o vinho biológico) com um modelo que apresenta um coeficiente de regressão não padronizado e padronizado de $\beta$ de 0,218 e 0,207, respetivamente (Tabela 5), sendo o coeficiente padronizado $\beta=0,207$ o coeficiente ajustado e o qual deve aparecer na equação de regressão linear múltipla.

\section{H 3. Quanto mais saudável for o estilo de vida do consumidor, mais favorável será a sua atitude com o vinho biológico.}

O modelo linear eliminou o fator Quanto mais saudável for o estilo de vida do consumidor, o que significa uma influência nula deste fator sobre a atitude para com o vinho biológico. Contudo, o facto de a H.3 não ter sido confirmada, indica não ser o estilo de vida saudável suficiente para induzir atitudes positivas pelo vinho biológico.

Estes resultados estão alinhados com os obtidos no estudo de Rojas-Méndez et al. (2015), onde, apesar de o fator consciência com a saúde ser determinante e ter poder preditivo sobre as atitudes positivas dos consumidores face ao vinho biológico, o fator estilo de vida saudável não tem uma relação direta nem poder preditivo sobre as atitudes face ao vinho biológico.

Tabela 5. Modelo de regressão linear para explicar a atitude face ao vinho biológico

$\begin{array}{cccccc}\text { Fator } & \text { Coef. Não padronizados } & \text { Coef. Padronizado } & \\ & \boldsymbol{\beta} & \mathrm{SE} & \boldsymbol{\beta} & \text { Valor } \mathbf{~} & \text { Sig (1-tailed). } \\ \text { Constante } & 2,440 & 0,206 & 11,820 & 0,000 \\ \begin{array}{c}\text { Consciência com } \\ \text { a saúde }\end{array} & 0,218 & 0,056 & 0,207 & 3,909 & 0,000 \\ & & \mathrm{R}^{2} 0,043 / \mathrm{R}^{2} \text { ajustado 0,040 }\end{array}$

Fator dependente: atitudes. / Fator estilo de vida saudável excluído

\subsubsection{Atitudes e sensibilidade ao preço}

Análise similar à anterior foi realizada com a finalidade de testar o poder preditivo dos fatores independentes atitude e sensibilidade ao preço, na intenção ou disposição face ao vinho biológico. 


\section{H 4. Quanto mais sensivel for um consumidor ao preço, menor disposição terá para o vinho biológico.}

Uma hipótese testada foi a da sensibilidade ao preço ser considerada um fator negativo na disposição face ao vinho biológico, não tendo esta hipótese (H.4) sido confirmada, em virtude de não atingir níveis significativos para explicar o efeito considerado, razão pela qual não faz parte do modelo. $O$ fator sensibilidade ao preço foi excluído do modelo linear.

\section{H 5. Quando a atitude do consumidor em relação ao vinho biológico é positiva, maior disposição terá face ao vinho biológico.}

Como resultado, encontrou-se a atitude face ao vinho biológico como o fator com maior correlação linear com a variável dependente (disposição face ao vinho biológico), com um coeficiente de regressão padronizado $\beta=0,375$. A hipótese H.5 foi confirmada no modelo obtido ao ter um valor de significância $p<0,05$ (Tabela 6).

Tabela 6. Modelo de regressão para explicar a disposição face ao vinho biológico

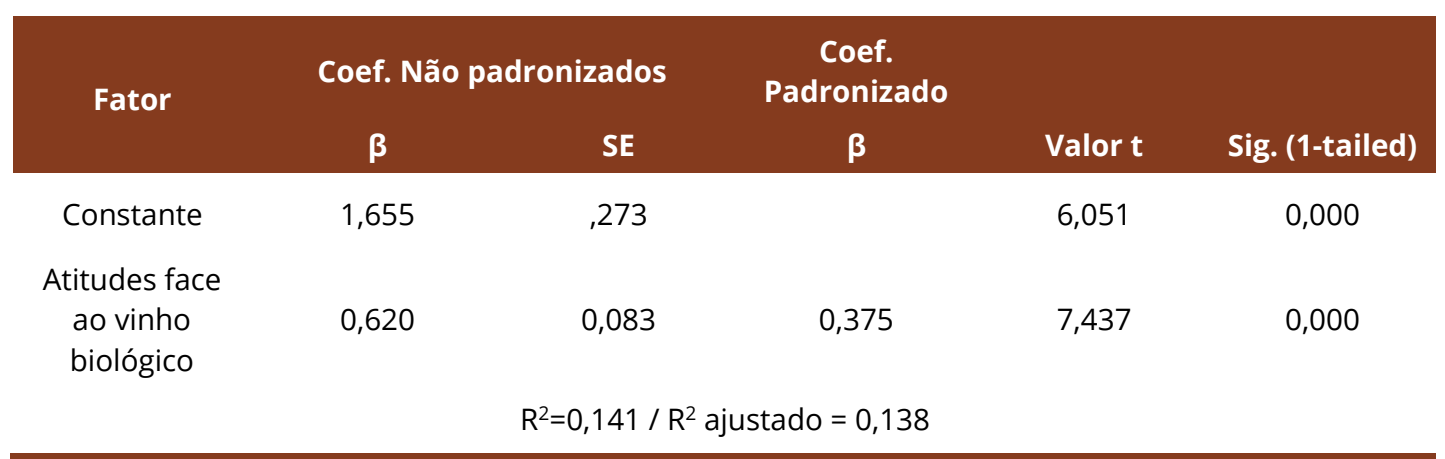

Sensibilidade ao preço foi excluído deste modelo

Ainda testada foi a hipótese de predição de maiores atitudes positivas levarem a uma maior disposição pelo vinho biológico. A H.5 foi confirmada (Tabela 6), indo ao encontro dos resultados encontrados pelos autores Rojas-Méndez et al. (2015) e Lee et al. (2014). Na literatura, embora o fator sensibilidade ao alto preço apareça como um fator negativo na disposição a comprar, consumir ou recomendar os produtos biológicos (Aertsens et al., 2009), na presente investigação, tal como no trabalho de Rojas-Méndez et al. (2015), esta não parece ser tão importante, já que os modelos de regressão não apresentam nenhum tipo de correlação linear.

Também nas duas regressões realizadas, não se detetou multicolinearidade (VIF $=1$ ), e se obtiveram valores perto de 2 no teste de Durbin-Watson, calculado para medir a existência de autocorrelação entre os resíduos, concluindo-se que não existe essa autocorrelação segundo a referência de Pestana \& Gageiro (2014).

\section{Conclusões}

Apesar de amplamente analisado na literatura, em Portugal não existem estudos do comportamento do consumidor sobre o vinho biológico que permitam perceber a situação e a evolução potencial do atual reduzido mercado dos vinhos biológicos. Este estudo, cujo objetivo foi o de compreender os fatores influenciadores do comportamento do consumidor na sua atitude e intenção de compra, consumo e recomendação do vinho biológico, é assim um modesto contributo para preencher esse vazio de conhecimento.

Dos resultados obtidos, inicialmente analisando os fatores a um nível descritivo e posteriormente, através de uma análise multivariada, conclui-se que, tendencialmente, o consumidor de vinho biológico apresenta boa perceção destes vinhos (sabor agradável, bom para saúde e produzido de forma amiga do ambiente). Estes consumidores também coincidem na importância da origem do vinho biológico (produzido em Portugal, conhecer a empresa vitivinícola produtora do vinho biológico e conhecer mais sobre este tipo de 
vinho). Também referem a qualidade desse vinho com um dos aspetos importantes a considerar. O consumidor de vinho biológico apresenta atitudes mais positivas em relação a este produto, comparativamente aos indivíduos não consumidores, mais neutrais. Também o facto de os consumidores de vinho biológico terem um estilo de vida saudável e maior consciência com a saúde, parece levar a uma maior propensão à compra e consumo. Em relação à sensibilidade ao preço, os não consumidores foram os que apresentaram a tendência a serem mais sensíveis. A partir das análises de regressão realizadas, conclui-se ser a perceção dos atributos do vinho biológico (ambientais, confiança, sabor-valor) um fator influenciador da previsão do comportamento dos consumidores de vinho biológico na sua disposição a comprar, consumir e recomendar. O sabor-valor é o fator com maior poder para explicar esse comportamento, seguido dos atributos ambientais associados ao vinho biológico, e da confiança dos consumidores nesse produto. Também foi possível caracterizar o consumidor em relação a aspetos de saúde e sensibilidade ao preço. Os consumidores de vinho e de vinho biológico, com consciência com a sua saúde, apresentam uma correlação positiva linear com atitude positiva face ao vinho biológico, de tal modo que, quanto maior for a atitude, maior será a sua disposição para comprar, consumir e recomendar vinho biológico, conclusão que converge com a de outros estudos na literatura. Os fatores estilo de vida saudável e sensibilidade ao preço não são determinantes das atitudes nem da disposição para comprar, consumir e recomendar o vinho biológico.

Finalmente, é de evidenciar que, apesar de ser difícil antecipar o comportamento do consumidor, pode inferir-se que fatores como a perceção, as atitudes e as características pessoais podem ajudar a prever o seu comportamento no mercado do vinho biológico, o que está alinhado com outros estudos em que estes e outros fatores foram testados em diferentes mercados, ficando plasmado que a construção teórica, as escalas de medição e os modelos explicativos do comportamento do consumidor usados são consistentes e ajustados ao encontrado na literatura. O outro, para evidenciar a necessidade de realizar mais estudos sobre o comportamento do consumidor, geradores de informação relevante para estabelecer novas propostas de marketing por parte dos responsáveis do setor, de modo a atrair aqueles que apenas têm um consumo ocasional (poucas vezes ao ano) e os não consumidores. Essa informação é chave para compreender o comportamento do consumidor, os seus hábitos de consumo e compra e as suas necessidades e constrangimentos, aspetos que podem ser impeditivos do alargamento do mercado português de vinho biológico. Essa informação é igualmente importante como suporte à tomada de decisão empresarial e ao desenvolvimento de planos e ações de marketing nesse mercado.

\section{Agradecimentos}

Os autores agradecem o suporte financeiro da Fundação para a Ciência e a Tecnologia (UID/04007/2020).

\section{Referências}

Aertsens, J., Verbeke, W., Mondelaers, K., \& Van Huylenbroeck, G. (2009). Personal determinants of organic food consumption: A review. British Food Journal, 111(2), 1140-1167.

Amato, M., Ballco, P., López-Galán, B., De Magistris, T., \& Vernau, F. (2017). Exploring consumers' perception and willingness to pay for "non-Added Sulphite" wines through experimental auctions: A case study in Italy and Spain. Wine Economics and Policy, 6, 146-154.

Asif, M., Xuhui, W., Nasiri, A., \& Ayyub, S. (2018). Determinant factors influencing organic food purchase intention and the moderating role of awareness: A comparative analysis. Food Quality and Preference, 63(18), 144-150.

Banco de Portugal (2017). Análise das empresas da indústria das bebidas. Estudos da Central de Balanços Janeiro. Recuperado em: novembro, 2018, de https://www.bportugal.pt/sites/default/files/anexos/pdf-boletim/estudos_da_cb_27_2017.pdf 
Barber, N., Taylor, C., \& Strick, S. (2009). Wine consumers' environmental knowledge and attitudes: Influence on willingness to purchase. International Journal of Wine Research, 1(1), 59-72.

Basha, M. B., Mason, C., Shamsudin, M. F., Hussain, H. I., \& Salem, M. A. (2015). Consumers attitude towards organic food. Procedia Economics and Finance, 31(5), 444-452.

Bonn, M. A., Cronin, J. J., \& Cho, M. (2016). Do environmental sustainable practices of organic wine suppliers affect consumers' behavioral intentions? The moderating role of trust. Cornell Hospitality Quarterly, 57(1), 21-37.

Cabo, P., Matos A., Fernandes, A. \& Ribeiro, M. I. (2016). Portugal biológico: Retrato da agricultura em modo de produção biológico em Portugal. In S. Fernández, D. Copena Rodríguez, \& D. Pérez Neira. Construíndo Conecemento Agroecolóxico: Cambiando os modelos de consumo para construír sistemas agroalimentarios sustentables (pp. 88-101) Vigo, Espanha: Grupo de Investigación há Economía Ecolóxica, Agroecoloxía e Historia.

Cardeira, R. F. (2009). Factores críticos de sucesso no mercado do vinho em Portugal e a Sustentabilidade do Sector Vitivinícola (Dissertação de mestrado). Instituto Superior Técnico, Lisboa, 2009. 101 p.

Centre for the Promotion of Imports - CPI. (2016). Market Information Database. Recuperado em: maio, 2019, de https://www.cbi.eu/sites/default/files/market_information/researches/product-factsheetorganic-wine-europe-wine-2013.pdf/

D'Amico, M., Di Vita, G., \& Monaco, L. (2016). Exploring environmental consciousness and consumer preferences for organic wines without sulfites. Journal of Cleaner Production, 120, 64-71.

Damásio, B. F. (2012). Uso da análise fatorial exploratória em psicologia. Avaliação Psicológica, 11(2), 213-228.

Daugbjerg, C., Smed, S., Andersen, L. M., \& Schvartzman, Y. (2014). Improving eco-labelling as an environmental policy instrument: Knowledge, trust and organic consumption. Journal of Environmental Policy and Planning, 16(4), 1-17.

Direcção Geral de Agricultura e Desenvolvimento Rural - DGADR. (2017). Estratégia Nacional para a Agricultura Biológica. Recuperado em: setembro 2018, de https://www.portugal.gov.pt /media/26727833/20170329-mafdr-estrategia-agriculturabiologica.pdf

Dziuban, C. D., \& Shirkey, E. S. (1974). When is a correlation matrix appropriate for factor analysis? Some decision rules. Psychological Bulletin, 81(6), 358-361.

Engel, J. F., Blackwell, R. D., \& Miniard, P. W. (1995). Consumer behavior (8th ed.). New York: Dryder.

Fotopoulos, C., Krystallis, A., \& Ness, M. (2003). Wine produced by organic grapes in Greece: Using means - End chains analysis to reveal organic buyers' purchasing motives in comparison to the non-buyers. Food Quality and Preference, 14(7), 549-566.

Gilinsky, A., Newton, S. K., \& Vega, R. F. (2016). Sustainability in the global wine industry: Concepts and cases. Agriculture and Agricultural Science Procedia, 8, 37-49.

Guia, A. (2014). O comportamento do consumidor de vinho no mercado Português (Tese de doutorado). Universidade de Trás-os-Montes e Alto Douro, Portugal.

Howard, J. A., \& Sheth, J. N. (1969). The theory of buyer behavior. In M. Baker. Marketing: Critical perspectives on business and management (Vol. 3, p. 71-84). London and New York: Routledge.

Instituto da Vinha e do Vinho - IVV. (2017). Vinhos e aguardentes de Portugal. Recuperado em: janeiro 2019, de https://www.ivv.gov.pt/np4/\%7B \$clientServletPath\%7D/?newsld=1736\&fileName $=I V V_{-}$WEB_TB.PDF

Jones, G. R., \& Hill, C. W. L. (2010). Theory of strategic management: with cases (9th ed.). China: Cengage Learning.

Kim, H., \& Bonn, M. A. (2015). The moderating effects of overall and organic wine knowledge on consumer behavioral intention. Scandinavian Journal of Hospitality and Tourism, 15(3), 295-310.

Kotler, P., \& Armstrongs, G. (2007). Marketing versión para Latinoamérica (11. ed.). Edo. de México: Pearson Educación de México, S.A.

Laroche, M., Bergeron, J., \& Barbaro-Forleo, G. (2001). Targeting consumers who are willing to pay more for environmentally friendly products. Journal of Consumer Marketing, 18, 503-520.

Le Douarin, S. (2016). La bio dans le monde. Montreuil, France. Recuperado em: julho 2019, de http://www.agencebio.org/sites/default/files/upload/documents/4_Chiffres/BrochureCC/carnet_mo nde_2016.pdf

Lee, W. F., Asgari, M., \& Siew, S. W. (2014). Price presentation effects on green purchase intentions. Journal of Product and Brand Management, 23(3), 230-239. 
Lucas, M. R. (2006). Handbook of consumer behaviour. Recuperado em: dezembro 2018, de http://agrimba.sggw.waw.pl/.

Mainardes, E. W., Araujo, D. V. B., Lasso, S., \& Andrade, D. M. (2017). Influences on the intention to buy organic food in an emerging market. Marketing Intelligence \& Planning, 35(7), 858-876.

Malhotra, N. K. (2011). Pesquisa de marketing (3a ed.). Sao Paulo: Pearson Prentice Hall.

Mann, S., Ferjani, A., \& Reissig, L. (2012). What matters to consumers of organic wine? British Food Journal, 114(2), 272-284.

Nicosia, F. M. (1966). Consumer decision processes: Marketing and advertising implications. Englewood, Cliffs, NJ: Prentice-Hall.

Nielsen. (2017). Vinhos crescem 8\% no on trade e 4\% do off trade. Recuperado em: fevereiro 2019, de http://www.nielsen.com/pt/pt/insights/news/2017/wines-grow-8-percent-in-on-trade-and-4percent-in-off-trade.html

Nielsen. (2018). Portugal com o maior crescimento em valor nos bens de grande consumo. Recuperado em: abril 2018, de http://www.nielsen.com/pt/pt/insights/news/2018/portugal-with-the-highest-growthin-value.

Nuttavuthisit, K., \& Thøgersen, J. (2017). The importance of consumer trust for the emergence of a market for green products: the case of organic food. Journal of Business Ethics, 140(2), 323-337.

Pestana, M. H., \& Gageiro, J. N. (2014). Análise de dados para ciências sociais: a complementaridade do SPSS (6a ed.). Lisboa: Edições Silabo.

Pink, M. (2015). The Sustainable wine market in Europe - Introduction to a market trend and its issues. Oeconomia, 14(2), 131-142.

Pivato, S., Misani, N., \& Tencati, A. (2008). The impact of corporate social responsibility on consumer trust: the case of organic food. Business Ethics, 17(1), 3-12.

Rodrigues, N. (2016). Comportamento do consumidor determinantes no consumo de alimentos biológicos (Dissertação de mestrado). Universidad de Coimbra, Portugal. 87 f.

Rojas-Méndez, J. I., Le Nestour, M., \& Rod, M. (2015). Understanding attitude and behavior of canadian consumers toward organic wine. Journal of Food Products Marketing, 21(4), 375-396.

Schäufele, I., \& Hamm, U. (2018). Organic wine purchase behaviour in Germany: Exploring the attitudebehaviour-gap with data from a household panel. Food Quality and Preference, 63(18), 1-11.

Schiffman, L., \& Kanuk, L. (2010). Comportamiento del Consumidor (10a ed.). México: Pearson Education.

Shafie, F. A., \& Rennie, D. (2012). Consumer perceptions towards organic food. Procedia: Social and Behavioral Sciences, 49, 360-367.

Shepherd, R., Magnusson, M., \& Sjöden, P. O. (2005). Determinants of consumer behavior related to organic foods. Ambio, 34, 352-359.

Sillani, S., Miccoli, A., \& Nassivera, F. (2017). Different preferences for wine communication. Wine Economics and Policy, 6(1), 28-39.

Silvério, M. (2000). Análise do mercado de vinho e das zonas vitivinícolas nacionais. Posicionamento, segmentação, preferências e atitudes. Caso particular: As sub-regiões do Alentejo (Tese de doutorado). Universidade de Évora, Portugal.

Steenkamp, J.-B. E. M. (1997). Dynamics in consumer behavior with respect to agricultural and food products. In B. Wierenga, A. van Tilburg, K. Grunert, J.-B E.M. Steenkamp, \& M. Wedel (Eds.), Agricultural marketing and consumer behavior in a changing world (pp. 143-188). Massachusetts: Kluwer Academic Publishers.

Stolz, H., \& Schmidt, O. (2008, June 16-20). Consumer attitudes and expectations of organic wine. In 16th IFOAM Organic World Congress. Modena, Italy.

Ueasangkomsate, P., \& Santiteerakul, S. (2016). A study of consumers' attitudes and intention to buy organic foods for sustainability. Procedia Environmental Sciences, 34, 423-430.

Weisstein, F.L., Asgari, M., \& Siew, S. W. (2014). Price presentation effects on green purchase intentions. Journal of Product and Brand Management, 23(3), 230-239.

Willer, H., \& Lernoud, J. (2017). The World of Organic Agriculture 2017. The World of Organic agriculture Statistics and Emerging Trends. Bonn, Alemanha: International Federation of Organic Agriculture Movements and Research Institute of Organic Agriculture.

Yadav, R., \& Pathak, G. S. (2016). Intention to purchase organic food among young consumers: Evidences from a developing nation. Appetite, 96(16), 122-128. 
Yin, S., Wu, L., Du, L., \& Chen, M. (2010). Consumers' purchase intention of organic food in China. Journal of the Science of Food and Agriculture, 90, 1361-1367.

Submetido em: $18 / 10 / 2019$
Aceito em: $19 / 05 / 2020$

Aceito em: 19/05/2020
Classificação JEL: M31 